\title{
Eight mutations including 5 novel ones in the COL1A1 gene in Czech patients with osteogenesis imperfecta
}

\author{
Lucie Hruskovaa ${ }^{a}$ Igor Fijalkowski ${ }^{b}$, Wim Van Hul ${ }^{b}$, Ivo Marik ${ }^{c, d}$, Geert Mortier ${ }^{b}$,Pavel Martasek ${ }^{a}$, Ivan Mazura ${ }^{a}$
}

Background and Aim. Osteogenesis imperfecta (OI), also called brittle bone disease, is a clinically and genetically heterogeneous disorder characterized by decreased bone density. Autosomal dominant forms result from mutations in either the COL1A1 (collagen type I alpha-1 chain) or COL1A2 (collagen type I alpha-2 chain) genes encoding the type I collagen. The aim of this study was to identify mutations and allelic variants of the COL $1 \mathrm{~A} 1$ gene in patients with osteogenesis imperfecta (OI).

Methods and Results. Molecular genetic analysis of the COL1A1 gene was performed in a cohort of 34 patients with OI. The DNA samples were analysed by PCR and Sanger sequencing. DNA changes in coding sequences of the gene were compared with Type 1 Collagen Mutation Database. Genetic variants resulting in either quantitatively or structurally defective protein production were found in 6 unrelated patients. Four identified mutations are connected to decreased protein production (Tyr47X, Arg131X, Arg415X, Gln1341X), 2 result in amino acid substitution (Cys61Phe, Pro1186Ala) and the last affects splicing (c.1057-1G>T). Further, one silent mutation (Gly794Gly) was detected. No protein analysis was performed.

Conclusion. Of the 8 identified mutations, 5 were novel and have not been reported before. Only one causes substitution of glycine located within the Gly-X-Y triplets in the triple helical domain. Two mutations are located in major ligand binding regions (MLBR) which are important for bone strength and flexibility. Although the genotype-phenotype correlation is still unclear, our findings should contribute to elucidating this relationship in patients diagnosed with Ol.

Key words: collagen type I, COL1A1, mutations, osteogenesis imperfecta

Received: November 9, 2015; Accepted: April 13, 2016; Available online: April 27, 2016 http://dx.doi.org/10.5507/bp.2016.022

${ }^{a}$ Department of Pediatrics and Adolescent Medicine, First Faculty of Medicine, Charles University in Prague, Czech Republic
bCentre of Medical Genetics, Antwerp University and University Hospital, Antwerp, Belgium
'Ambulant Centre for Defects of Locomotor Apparatus 1.1.c., Prague, Czech Republic
dFaculty of Medical Studies, West Bohemia University, Pilsen, Czech Republic
Corresponding author: Lucie Hruskova, e-mail:black.luca@seznam.cz

\section{INTRODUCTION}

Osteogenesis imperfecta $(\mathrm{OI})$ is a disorder of human connective tissue characterized by low bone mass and decreased bone material strength resulting in a higher risk of fractures. Affected patients show variable clinical signs such as blue sclerae, hearing loss, joint hypermobility, skin hyperlaxity, normal to low stature and dentinogenesis imperfecta (DI). Skeletal radiographs reveal osteopenia and pathological fractures of long bones and/ or vertebrae. Based on clinical and genetic features fourteen types of the disease (type I to XIV) have been delineated to date. Depending on the genetic basis, two forms of OI - collagenous (types I to IV) and non-collagenous (types V to XIV), are described ${ }^{1}$. About $90 \%$ of OI cases are due to dominant mutations in either the COL1A1 (collagen type I alpha-1 chain) gene or COL1A2 (collagen type I alpha-2 chain) gene both of which encode the type I collagen protein. These mutations result in either reduced production (haploinsufficiency) of the protein or in the synthesis of structurally abnormal collagen ${ }^{1,2}$. Haploinsufficiency results from mutations creating stopcodons (premature termination codon) indicating transcription termination of affected gene. Such shortened alpha chains miss C-propeptide domain which is important for alpha chains assembling into triple helix and are destroyed by a process called nonsense-mediated decay. This process eliminates mutant alpha chains and leads to the synthesis of reduced amount of normal protein. Decreased production (haploinsufficiency) of collagen type I is typical for OI type I (ref. ${ }^{1,3,4}$ ). Patients affected by OI type I (Dominantly inherited OI with blue sclerae) are of normal stature and have blue sclera (probability of blue sclerae occurrence is near to $100 \%\left(\right.$ ref. $\left.^{5}\right)$ ). Bone fractures occur especially during childhood and moderate bone deformities, especially of the lower limbs and spine, could be observed. Other clinical sign observed in OI type I individuals is hearing impairment which usually results in hearing loss ${ }^{4}$. Based on presence of dental abnormalities, type I is divided into A and B subtypes (with and without dentinogenesis imperfecta, respectively) (ref. $\left.{ }^{2,6}\right)$. In general, patients affected with OI type IA are of normal stature and have lower incidence of fractures whereas OI type IB patients have higher frequency of fractures and more skeletal deformities resulting in shorter stature ${ }^{5,7}$. Synthesis of abnormal collagen type I is described in OI types II, III and IV. Mutations affecting the structure of alpha chains include amino acids substitutions, exon-skip- 
ping mutations and more complex gene rearrangements ${ }^{3}$. OI type II (Lethal perinatal OI with radiographically crumpled femora and beaded ribs) is a lethal type of OI with high percentage of perinatal mortality. In rare cases, patients survive few days. Typical clinical signs include multiple intrauterine fractures, severe skeletal deformities, blue sclera, low birthweight and low birth crown-heel length ${ }^{8,9}$. The third type of OI (Progressively deforming OI with normal sclerae) is the most severe form characterised by progressive deformities, high frequency of fractures, short stature due to severe skeletal deformities, triangular face, hearing impairment, basilar impression and dentinogenesis imperfecta. In some cases, infants die due to cardiac or pulmonary insufficiency ${ }^{8,9}$. The last type of OI with origin in defective collagen type I production, OI type IV (Dominantly inherited OI with normal sclerae), is the most heterogeneous form of the disease. Individuals exhibit mild to moderate phenotype and are of short stature. First fractures occur at birth, moderate deformities (especially of long bones of the lower and upper limbs) are presented. Teeth and hearing impairment could be presented ${ }^{5}$. Like the first OI type, subtypes A and B (with and without DI, respectively) are distinguished ${ }^{2,6}$. In the remaining $10 \%$ of cases, OI is the result of recessive mutations in IFITM5 (Interferon-induced transmembrane protein 5, OI type V; bone formation co-participant) (ref. ${ }^{10}$ ), SERPINF1 (Serpin peptidase inhibitor, clade F, member 1, OI type VI; bone mineralization participation) (ref. ${ }^{11}$ ), CRTAP (Cartilage associated protein, OI type VII; 3-hydroxylation complex component) ( ref. $^{12}$ ), P3H1 (Prolyl 3-Hydroxylase 1, OI type VIII; 3-hydroxylation complex component) (ref. ${ }^{12,13}$ ), PPIB (Peptidyl-prolyl isomerase 1 (Cyclophylin B), OI type IX; 3-hydroxylation complex component) (ref. ${ }^{14}$ ), SERPINH1 (Serpin peptidase inhibitor, clade $\mathrm{H}$, member 1 , OI type X; collagen type I chaperone) (ref. ${ }^{12}$ ), FKBP10 (FK506-binding protein 10, OI type XI; collagen type I chaperone) ( ref. $^{12}$ ), SP7 (Osteoblast-specific transcription factor SP7, OI type XII; cell differentiation coparticipant) (ref. ${ }^{11}$ ), BMP1 (Bone morphogenetic protein 1, OI type XIII; embryogenesis and skeletogenesis process co-participant) (ref. ${ }^{11,15}$ ) or TMEM38B (Transmembrane protein 38B, OI type XIV; cell differentiation co-participant) (ref. ${ }^{11}$ ). Recently, mutations in the WNT1 gene (Winglesstype MMTV integration site family, member 1, bone mass regulator) have been described in patients diagnosed with OI type IV (ref. $\left.{ }^{16}\right)$.

The incidence of OI ranges from 1:25000-40000 (nonlethal forms) to 1:50000 (lethal forms) live births in the world $^{17}$. Currently, no data are available on OI prevalence in the Czech population.

The aim of this study is identification of mutations and allelic variants of COL1A1 gene. Type I collagen is a heterotrimer, composed of two alpha-1(I) and one alpha-2(I) chain encoded by COL1A1 and COL1A2 respectively. Alpha-1(I) chain represents $2 / 3$ of this heterotrimer ${ }^{1}$. Generally, in the random selection of abnormal chains $75 \%$ of mutant heterotrimers contain one or more defective alpha-1(I) chains ${ }^{4}$. Further, possible genotypephenotype relationship of identified mutations will be discussed. Determination of possible effect of identified mutations on clinical picture of affected patients could be helpful for providing well-timed and helpful treatment of affected individuals.

\section{MATERIALS AND METHODS}

\section{Material}

Included in the study were 34 patients with a diagnosis of OI. There were 11 males and 23 females aged 7 to 57 years. The diagnosis was based on evaluation of clinical, radiological and biochemical features. In 19 patients, a diagnosis of OI type IA was made, 6 individuals were affected by type III, in 4 cases OI type IVA was identified and 5 patients suffered from type IVB. No case of OI type II was included in the study.

This study was performed in accordance with principles of the Declaration of Helsinki and approved by the Ethics Committee of General University Hospital in Prague (project 83/14). Participants provided their written informed consent with involvement in the study. The purpose and procedures of the research were explained, rights to refrain from the study under any circumstances and for any reason were emphasized. Informed consent was ensured by the Ambulant Centre for Defects of Locomotor Apparatus in Prague and was carried out according to "Informed approbation in medical genetics" by the committee of the Society of Medical Genetics (SMG) Czech medical Association of J. E. Purkyne.

\section{Laboratory methods}

Genetic analysis was restricted to the coding (and flanking non-coding) sequences (51 exons) of the COL1A1 gene whose product (alpha-1(I) chain) is two copies in the collagen type I (ref. ${ }^{18}$ ). Genomic DNA was extracted from peripheral blood leukocytes using a standard extraction method (QIAamp DNA Blood Midi Kit, QIAGEN), followed by high resolution melting analysis (HRM), polymerase chain reaction (PCR) amplification and Sanger sequencing. PCR amplification was performed using 47 sets of forward and reverse primers located in sequences of flanking introns (primer sequences are available on request). PCR products were confirmed by horizontal gel electrophoresis. Sequences of these PCR products were obtained on the ABI PRISM 3130xl Genetic Analyzer automated sequencer (Applied Biosystems, Foster City, CA, USA) using the BigDye terminator cycle sequencing chemistry. The data were compared to the wild-type sequence as submitted to Ensembl accession no. ENST00000225964. Nucleotides were numbered from the first base of the start codon (ATG) of the cDNA reference sequence according to GenBank NM_000088.3. Amino-acid residues were numbered from the start codon for methionine (ATG) of the alpha-1 chain of type I collagen (reference sequence GenBank NM_000079.2).

Novel mutations were identified by their absence from the Osteogenesis Imperfecta Variant Database, collagen, type I, alpha 1 (COL1A1) (https://oi.gene.le.ac. uk/home.php?select_db=COL1A1) and the Osteogenesis 
Imperfecta Variant Database, collagen, type I, alpha 2 (COL1A2) (https://oi.gene.le.ac.uk/home.php?select $\mathrm{db}=\mathrm{COL} 1 \mathrm{~A} 2)$. Possible splice site effect of novel intronic mutation was evaluated using the PC program Alamut (used algorithms: SpliceSiteFinder, MaxEntScan, NNSPLICE, GeneSplicer, Human Splicing Finder).

\section{RESULTS}

We identified COL1A1 mutations of coding and non-coding sequences in $7 / 34$ patients. None of these mutations were found in the DNA of a healthy control - Caucasian population. Four of the mutations result in premature stopcodon (c.141C>A p.Tyr47X, c. $391 \mathrm{C}>\mathrm{T}$ p.Arg131X, c. $1243 \mathrm{C}>\mathrm{T}$ p.Arg415X, c.4021C $>\mathrm{T}$ p.Gln 1341X), two are missense mutations (c.182G>T p.Cys61Phe, c.182G>T p.Pro1186Ala), one is located in intronic sequence (c.1057-1G>T) and the last one is silent mutation of glycine at position 794 of the COL1A1 gene (Table 1, Fig. 1).

The first patient (OI17) in this study was a 23-year-old woman affected by OI type IA. She is of subnormal height and suffers from joint hypermobility. In this case, we identified the heterozygous p.Tyr47X mutation in COL1A1.

The second case (OI23) was a 45-year-old man of unaffected parents diagnosed with OI type III. He had had multiple fractures since childhood, especially of the

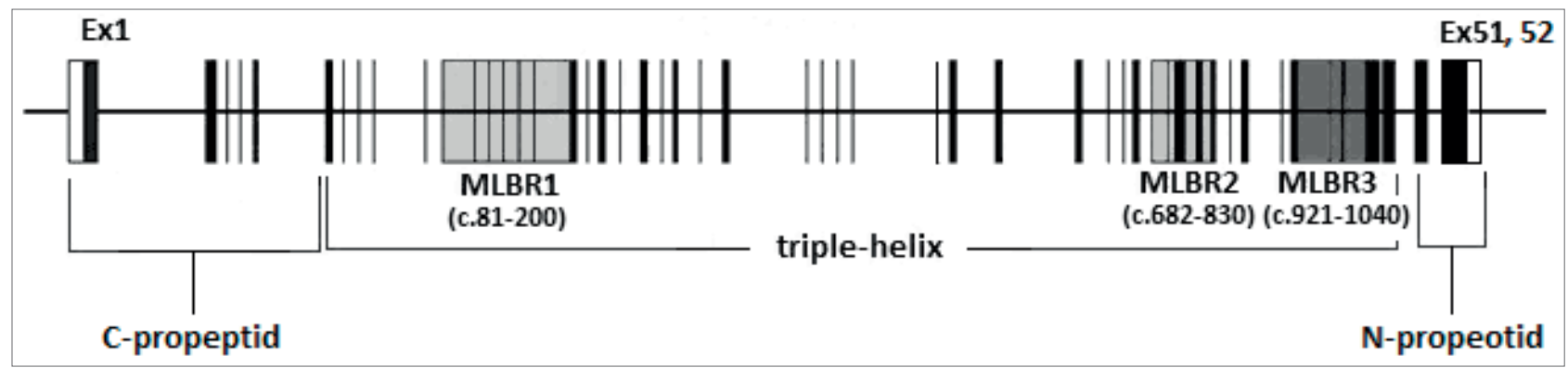

Fig. 1. Localization of identified COL1A1 gene mutations. The exons are presented by vertical lines. Multi Ligand Binding Regions are represented by boxes in shades of grey.

Table 1. Basic clinical and personal data and genetic findings of osteogenesis imperfecta (OI) probands with COL1A1 mutations.

\begin{tabular}{|c|c|c|c|c|c|c|c|}
\hline Nucleotide change ${ }^{\mathrm{I}}$ & c. $141 \mathrm{C}>\mathrm{A}$ & c. $182 \mathrm{G}>\mathrm{T}$ & c. $391 \mathrm{C}>\mathrm{T}$ & $\begin{array}{c}\text { c. } 1243 C>T \\
\text { c. } 3556 \mathrm{C}>\mathrm{G}\end{array}$ & c. $4021 \mathrm{C}>\mathrm{T}$ & c. $1057-1 G>T$ & c. $2382 \mathrm{~T}>\mathrm{C}$ \\
\hline $\begin{array}{l}\text { Predicted amino } \\
\text { acid change }{ }^{I I}\end{array}$ & Tyr47X & Cys61Phe & $\operatorname{Arg} 131 X$ & $\begin{array}{c}\text { Arg415X } \\
\text { Pro1186Ala }\end{array}$ & Gln 1341X & $\begin{array}{l}\text { splice-site } \\
\text { mutation }\end{array}$ & Gly794Gly \\
\hline $\mathrm{HR} / \mathrm{HM}^{\mathrm{III}}$ & HR & HM & HR & HR HR & HR & HR & HR \\
\hline Protein defect & quantitative & qualitative & quantitative & $\begin{array}{l}\text { quantitative } \\
\text { qualitative }\end{array}$ & quantitative & qualitative & $\begin{array}{c}\text { silent } \\
\text { mutation }\end{array}$ \\
\hline SNP property ${ }^{\mathrm{I}}$ & Exon 2 & Exon 2 & Exon 5 & $\begin{array}{l}\text { Exon } 19 \\
\text { Exon } 48\end{array}$ & Exon 50 & Intron 16 & Exon 34 \\
\hline Novel & Yes & No & No & $\begin{array}{l}\text { Yes } \\
\text { No }\end{array}$ & Yes & Yes & Yes \\
\hline OI patient & OI17 & $\mathrm{OI} 23$ & OI42 & OI14 & $\mathrm{OI} 2$ & OI21 & OI30 \\
\hline $\mathrm{Sex}^{\mathrm{IV}}$ & $\mathrm{F}$ & M & $\mathrm{F}$ & $\mathrm{M}$ & $\mathrm{M}$ & M & $\mathrm{F}$ \\
\hline Age (years) & 23 & 45 & 24 & 22 & 18 & 50 & 24 \\
\hline Type of OI & IA & III & IA & IA & IA & IA/IVA & IVA \\
\hline $\mathrm{S} / \mathrm{F}^{\mathrm{V}}$ & $\mathrm{S}$ & S & $\mathrm{F}$ & $\mathrm{S}$ & $\mathrm{S}$ & $\mathrm{F}$ & $\mathrm{S}$ \\
\hline Blue sclera $^{\mathrm{VI}}$ & - & - & - & + & + & + & $+1-$ \\
\hline Hearing loss ${ }^{\mathrm{VI}}$ & - & - & - & - & - & + & - \\
\hline Bone deformities $^{\mathrm{VI}}$ & - & + & + & + & + & + & + \\
\hline
\end{tabular}

IThe numbering for the nucleotide changes and exons are based on cDNA sequence in accordance with GenBank entry NM_000088.3.

${ }^{\text {II }}$ Numbered with reference to the A of initiation ATG as 1 according to the reference sequence NP_000079.2.

${ }^{\text {III }} \mathrm{HR}$ : heterozygous allele state; HM: homozygous allele state

${ }^{\mathrm{IV}} \mathrm{M}$ : male; F: female

${ }^{v} \mathrm{~F}$ : familial OI case; $\mathrm{S}$ : sporadic OI case

$\mathrm{VI}_{+}$: presence of clinical sign; -: absence of clinical sign; +/-: clinical sign changed with age; $\mathrm{x}$ : no data 
lower limbs, and is confined to a wheelchair. He has a barrel-shaped chest, severe bone deformities and muscle weakness in the lower limbs. We identified homozygous substitution of cysteine by phenylalanine at position p.61 in the DNA sample of this individual. This mutation from the program Alamut was predicted as disease causing $(P$ $=1.0$ ).

The third case (OI42) was a 24-year-old woman suffering from OI type IA. She is of subnormal height and has a mild barrel-shaped chest. DNA analysis identified a premature stopcodon at residue p.131 coding for arginine. She has four other affected relatives; unfortunately their DNA samples were not available.

The next case was a 22-year-old young man (OI14) suffering from OI type IA. He shows blue sclerae, has a relatively short barrel-shaped chest and mild deformation of the lower limbs and vertebrae. In this patient, we identified a heterozygous p.Arg415X mutation in exon 19. Even though it is represented in a sequence database (rs72648326), it is disease causing due to production of shortened alpha chains with loss of heterotrimer formation function. Further, a substitution of proline by alanine at position p.1186 was also identified in heterozygous state. This mutation, using the program Alamut, was predicted as disease causing $(P=0.998)$.

The patient with an identified mutation in the coding sequence of the COL1A1 gene, p.Gly1341X in heterozygous state, was an 18-year-old boy (OI2) affected by OI IA. The boy is of subnormal height. He has light blue sclerae, barrel-shaped chest and muscle hypotrophy of the limbs. He had suffered from multiple fractures of the spine and both upper and lower limbs.

The heterozygous change c.1057-1G>T was identified in the DNA of a 50-year-old man (OI21) with clinical phenotype corresponding with type IA/IVA of the disease. He suffered from multiple fractures of the limbs since childhood. He has blue sclerae, deformed bones and suffers from hearing loss.

Further, genetic analyses of the 34 patients identified 38 polymorphisms in the COL1A1 gene. This also includes a silent heterozygous variant of glycine at position p.794 of the COL1A1 gene identified in the case of a 24-year-old woman (OI30) diagnosed with OI IVA. She suffered multiple fractures of the lower limbs until the age of 18. The patient is of subnormal height, has deformed lower limb bones and weak muscles. She also showed joint hypermobility. The sclera had been light blue after the birth but had whitened during childhood. This patient was treated with bisphosphonates until the age of 21. Nine of 38 identified polymorphisms were situated in coding sequence, 7 were localised in MLBRs and one occurred in 3 ' untranslated region of the gene. Allele frequencies of polymorphisms identified in OI patients correlate with Population genetics available on Ensembl database (http://www.ensembl.org/Homo_sapiens/Transcript/ Variation_Transcript/Table?db=core;g=ENSG000001088 21;r=17:50183289-50201632;t=ENST00000225964).

\section{DISCUSSION}

All of the identified mutations resulting in decreased production of collagen type I (c.141C>A p.Tyr47X, c.391C > T p.Arg131X, c.1243C>T p.Arg415X, c.4021C>T p.Gln $1341 \mathrm{X}$ ) were found in patients affected by OI type I. Except one case (p.Arg131X, OI42), it was the first occurrence of the disease in the family history. Furthermore, in all cases we were able to observe one common clinical sign - subnormal body height. Only the Arg131X had been described in the case of Italian patients with a positive OI family history (the mutant allele was also detected in the mother of the individual). Unfortunately, the clinical data on this individual are not available ${ }^{19}$.

The nucleotide change c.182G $>$ T results in substitution p.Cys61Phe in the COL1A1 gene. The identified mutation is in a homozygous state and is localized on the second exon of the COL1A1 gene which is a part of the N-terminal propeptide domain of the pro-alpha1(I) collagen chain encoded by the first five exons and by a part of the sixth exon of the gene ${ }^{3}$. The integrity of N-propeptide is important for proper chain association and for subsequent folding of individual alpha chains into a collagen trimer with triple helical configuration (the importance of structure and folding of triple helix is also true for C-propeptide). In this process, cysteine residues play a major role through formation of intra- and interchain disulphide bonds. During proteosynthesis, the $\mathrm{N}$-terminal propeptide is removed from the pro-alpha-1(I) chain by specific proteinase to form the alpha-1(I) chain terminated by N-telopeptide. Because the substitution is not localized close to the N-proteinase cleavage site, its occurrence is not likely to prevent $\mathrm{N}$-propeptide removal ${ }^{20}$. The substitution is also localized in the von Willebrand factor type $\mathrm{C}$ (vWF-C) binding region defined by amino acid residues 38-96 (ref. ${ }^{21}$ ). Although the mutation could delay vWF-C binding, it is unknown whether or how it affects collagen type I processing. This mutation was first described by Zhang et al. (2012) in the case of a 17-yearold Chinese boy affected by the OI type I (first occurrence of the disease in the family, allelic variant not described). Clinical features described in this case were blue sclera and multiple fractures (more then 10) $\left(\right.$ ref. $\left.^{13}\right)$. We found this change in a homozygous state in the patient diagnosed with the progressively deforming form of OI (type III). Individuals have soft, fragile, deformed bones and may suffer from neurologic and respiratory complications. The other clinical picture includes severe scoliosis, blue colour of sclera, hearing loss and dentinogenesis imperfecta $^{22}$. The only clinical sign described in both cases with identified p.Cys61Phe substitution was high frequency of fractures.

Substitution of proline by alanine at position p.1186 of the COL1A1 protein is described in a case identified as OI type IA. The mutation is localized in exon 48 which is part of the third important ligand binding region, called the Major Ligand Binding Region (MLBR). There are three such regions (MLBR1-3) defined by codons 81-200 (MLBR1), 682-830 (MLBR2) and 920-1040 (MLBR3) (codon numbers start at the first amino acid residue of the 
helical domain of alpha chains) (ref. $\left.{ }^{18,23}\right)$. These regions produce intermolecular binding of collagen type I with other connective tissue proteins like integrins, COMP (cartilage oligomeric matrix protein), serpinH1, phosphophoryn and others. These interactions result in increasing bone strength and elasticity ${ }^{18}$. The MLBR3 region is encoded by a part of exon 46, the entire exons 47 and 48 and part of the exon 49 sequence $^{23}$. Concretely, this region including Pro1186 of the collagen type I molecule, binds amyloid beta A4 precursor protein (APP) that mediates cell-cell interactions ${ }^{18}$. It is also suggested that amyloid precursor protein is implicated in the regulation of synapse formation, neural plasticity and iron export ${ }^{24-26}$. Other molecules bound to this part of the alpha-1(I) chain are interleukin 2 (IL2; regulates activity of T-lymphocytes) (ref. ${ }^{18,27}$ ) and the collagen binding factor ENDO180 (Mannose receptor, C-type, 2; MRC2) which acts as collagen-binding receptor in the process of endocytosis. It may also play a role in the catabolism of extracellular matrix collagens. Finally, it is suggested this protein may participate in cell-matrix adhesion and cell migration ${ }^{18,28}$. The identified mutation disrupts the binding sequence. A further aspect is that p.Pro1186Ala substitution could negatively affect the stability of collagen type I. Proline and hydroxyproline residues are important amino acids representing 2/3 of all the $\mathrm{X}$ and $\mathrm{Y}$ positions of the Gly-X-Y motive in alpha chains. High frequency of these two amino acids increases the stability of molecules due to creation of interactions within their side chains and other triple helices ${ }^{29}$. There is evidence that substitution of proline localized within the triple helical domain of the collagen protein affects thermal stability of the collagen fibril since denaturation temperatures increase with increasing content of proline and hydroxyproline residues $^{30}$. This variant was also predicted to be damaging using algorithms MutationTester and SIFT.

Mutation in the intronic sequences was identified in one patient. Transversion $\mathrm{G}$ to $\mathrm{T}$ at position c.1057-1 in the COL1A1 gene was found in a man affected by OI type IVA. Nucleotide change is situated in the acceptor splice site of exon 17 and has been predicted to create a new acceptor splice site localized one base downstream to the first nucleotide of the first codon for glycine (GGT) of the exon 17. Consequently there is a shift of the reading frame of the exon 17 what is completely included in the MLBR1 $\left(\right.$ ref. $\left.{ }^{18}\right)$. Several extracellular matrix molecules are known to bind to this part of collagen type I. These include interleukin 2 (regulation of lymphocytes activity), $\alpha 2 \beta 1$ integrin which is able to bind other molecules of extracellular matrix (ECM) and GE-decorin (guanidine-extracted decorin) that appears to influence fibrillogenesis and interacts with other molecules of ECM (ref. ${ }^{18,27,31,32}$ ). Further, there are binding sites for osteonectin - a sodium binding glycoprotein that increases bone mineralization, and serpinH1 protein which ensures thermal stabilization of the triple helix domain during procollagen synthesis, helps the folding and assembly of procollagen molecules and participates in the transport of structurally unaffected molecules from the endoplasmic reticulum ${ }^{18,33,34}$. The c.1057-1G>T mutation results in production of structurally defective collagen type I with delayed binding ability of other ECM molecules increasing bone stability.
The silent c.Gly794Gly variant was identified in the case of a patient affected by OI type IVA. Even though, it is situated in the COMP binding domain defined by codons 582 to 638 , the variant does not alter the reading frame and so does not affect the protein production ${ }^{18}$.

This study is the first comprehensive analysis of patients affected by the collagenous forms of the osteogenesis imperfecta disease. COL1A1 mutations were only found in $20.6 \%$ of analysed individuals. The low percentage could be explained by the disease causing mutations occurring in other genes, like COL1A2, PPIB, CRTAP and others, involved in collagen type I processing and synthesis, by the lower probability of detecting $\mathrm{C} / \mathrm{G}$ and $\mathrm{A} / \mathrm{T}$ single nucleotide polymorphisms and small homozygous insertions/deletions shift using the HRM method as these may be very close in Tm to the wild type. For some exons, it can also be difficult to interpret HRM results because of the presence of several mutations or polymorphisms ${ }^{35}$. Additional functional experiments need to be performed to evaluate the effect of identified mutations. Further, analyses of COL1A1coding sequences excluded by HRM will be prepared.

Currently, COL1A2 genetic screening is performed to provide additional variants in patients diagnosed with one of dominant OI types. 46 of 52 coding (and flanking non-coding) sequences of the gene were analysed. Disease causing mutation of exon 40 (p.Gly814Trp) was identified in a patient affected by the third form of $\mathrm{OI}$ $\left(\right.$ ref. $\left.^{36}\right)$. Further, genetic analyses identified 14 single nucleotide polymorphisms. 4 were in coding sequence, 1 occurred in MLBR3. Allele frequencies of polymorphisms identified in COL1A2 gene correlate with Population genetics available on Ensembl (http://www.ensembl. org/Homo_sapiens/Transcript/Variation_Transcript/ Table? $\mathrm{db}=$ core;g=ENSG00000164692;r=7:9439456194431232;t=ENST00000297268).

\section{CONCLUSION}

Novel candidate COL1A1 mutations were identified in patients diagnosed with dominant OI forms. Moleculargenetic analyses of another 6/52 COL2A1 coding sequences are currently being prepared. Analyses of other genes involved in the disease origin are also planned as mutations in these genes may account for autosomal recessive forms in the OI patients involved in the study. It could be also helpful to analyse mRNA in patients with identified non-coding sequences mutations to define the effect of DNA splicing and collagen type I production.

Acknowledgement: We are indebted to all patients for their interest and cooperation.

This study was supported by the grants SVV-2013266517, SVV-2014-260034, SVV-2015-260158, PRVOUK P24/1LF/3 and UNCE 204011 from the Charles University in Prague.

Author contributions: LH: data analysis and interpretation, literature search, manuscript writing; IF, WVH, GM: data analysis and interpretation; IM: clinical data col- 
lection and interpretation; PM: data interpretation; IM: study design, data analysis and interpretation.

Conflict of interest statement: The authors state that there are no conflicts of interest in this study regarding the publication of this article.

\section{REFERENCES}

1. Forlino A, Cabral WA, Barnes AV, Marini JC. 2011. New perspectives on osteogenesis imperfecta. Nat Rev Endocrinol 2011;7:540-57.

2. Endotext [homepage on the Internet]. MDText.com, Inc. Available from: http://www.endotext.org/chapter/osteogenesis-imperfecta/7/. Accessed April 15, 2015. Accessed June 16, 2014.

3. Dalgleish R. The human type I collagen mutation database. Nucleic Acids Res 1997;25:181-7.

4. Bateman JF, Boot-Handford RP, Lamandé SR. Genetic diseases of connective tissues: cellular and extracellular effects of ECM mutations. Nat Rev Gen 2009;10:173-83.

5. Van Dijk FS, Sillence DO. Osteogenesis imperfecta: clinical diagnosis, nomenclature and severity assessment. Am J Med Genet Part A 2014;164A:1470-81.

6. Marini JC, Rajpar MH. Osteogenesis imperfecta. In: Thakker RV White MP, Eisman JA, Igarashi T, editors. Genetics of Bone Biology and Skeletal Disease. New York: Academic Press 2013; 257-73.

7. Paterson CR, McAllion S, Miller R. Heterogeneity of osteogenesis imperfecta type I. J Med Genet 1983;20:203-5.

8. Sillence DO, Senn A, Danks MD. Genetic heterogenity in osteogenesis imperfecta. J Med Genet 1979;16:101-16.

9. Roughley PJ, Rauch F, Glorieux FH. Osteogenesis imperfecta - clinical and molecular diversity. European Cells and Materials 2003;5:41-7.

10. Cho T-J, Lee K-E, Lee S-K, Song SJ, Kim KJ, Jeon D, Lee G, Kim H-N Lee HR, Eom H-H, Lee Z-H, Kim O-H, Park W-Y, Park SS, Ikegawa S, Yoo WJ, Choi IH, Kim J-W. A single recurrent mutation in the 50-UTR of IFITM5 causes osteogenesis imperfecta type V. Am J Hum Genet 2012;91:343-8.

11. Shaheen R, Alazami AM, Alshammari MJ, Faqeih E, Alhashmi N, Mousa N, Alsinani A, Ansari S, Alzahrani. F, Al-Owain M, Alzayed ZS Alkuraya, FS. Study of autosomal recessive osteogenesis imperfecta in Arabia reveals a novel locus defined by TMEM38B mutation. J Med Genet 2012;49:630-5.

12. Lapunzina $P$, Aglan M, Temtamy S, Caparrós-Martín JA, Valencia M Letón R, Martínez-Glez V, Elhossini R, Amr K, Vilaboa N, Ruiz-Perez VL. Identification of a frameshift mutation in Osterix in a patient with recessive osteogenesis imperfecta. Am J Hum Genet 2010;87:110-4

13. Zhang ZL, Zhang H, Ke Y, Yue H, Xiao WJ, Yu JB, Gu JM, Hu WW, Wang $\mathrm{Ch}, \mathrm{He}$ JW, Fu WZ. The identification of novel mutations in COL1A1, COL1A2, and LEPRE1 genes in Chinese patients with osteogenesis imperfecta. J Bone Miner Metab 2012;30:69-77.

14. Cundy T. Recent advances in osteogenesis imperfecta. Calcif Tissue Int 2012;90:439-49.

15. Martinéz-Glez V, Valencia M, Caparrós-Martín JA, Aglan M, Temtamy S, Tenorio J, Pulido V, Lindert U, Rohrbach M, Eyre D, Giunta C, Lapunzina P, Ruiz-Perez VL. Identification of a mutation causing deficient BMP1/mTLD proteolytic activity in autosomal recessive osteogenesis imperfecta. Hum Mutat 2012;33:343-50.

16. Fahiminiya S, Majewski J, Mort J, Moffatt P, Glorieux FH, Rauch F. Mutations in WNT1 are a cause of osteogenesis imperfecta. J Med Genet 2013;50:345-8.

17. Hudaková-Maříková O, Mařík I, Zemková D, Šedová M, Mazura I, Kuklík M. Osteogenesis imperfecta from the point of view clinical and anthropological characteristics of the disease and differential diagnostics of its types. Pohybové ústrojí 2007;14(3-4):325-9.

18. Sweeney, SM, Orgel JP, Fertala A, McAuliffe JD, Turner KR, Di Lullo GA, Chen S, Antipova O, Perumal S, Ala-Kokko L, Forlino A, Cabral
WA, Barnes AM, Marini JC, San Antonio JD. Candidate cell and matrix interaction domains on the collagen fibril, the predominant protein of vertebrates. J Biol Chem 2008;283:21187-97.

19. University of Leicester. Osteogenesis Imperfecta Variant Database (2008). https://oi.gene.le.ac.uk/variants.php?select_db=COL1A1\&a ction=view\&view=0001207\%2C0000492\%2C21\&order=Variant $\% 2$ FProtein\%2CASC. Accessed 5 March 2013.

20. Hulmes DJ. Building collagen molecules, fibrils and suprafibrillar structures. J Struct Biol 2002;137:2-10.

21. UniProt Knowledgebase. http://www.uniprot.org/uniprot/ P02452\#family_and_domains

22. Sorin H, Cornel C, Cristian CG, Iuliana P. Osteogenesis imperfecta: forensic assessment of traumatic injuries. Case report and literature review. Rom J Leg Med 2008;16:275-82.

23. Marini JC, Forlino A., Cabral WA, Barnes AM, San Antonio JD, Milgrom S, Hyland JC, Körkkö J, Prockop DJ, De Paepa A, Coucke P, Symoens S, Glorieux FH, Roughley PJ, Lund AM, Kuurila-Svahn K, Hartikka H, Cohn DH, Krakow D, Mottes M, Schwarze U, Chen D, Yang K, Kuslich Ch, Troendle J, Dalgleish R, Byers PH. Consortium for osteogenesis imperfecta mutations in the helical domain of type I collagen: regions rich in lethal mutations align with collagen binding sites for integrins and proteoglycans. Hum Mutat 2007;28:209-21.

24. Duce JA, Tsatsanis A, Cater MA, James SA, Robb E, Wikhe K, Leong SL, Perez K, Johanssen T, Greenough MA, Cho HH, Galatis D, Moir RD, Masters CL, McLean C Tanzi RE, Cappai R, Barnham KJ, Ciccotosto $\mathrm{GD}$, Rogers JT, Bush Al. Iron-export ferroxidase activity of $\beta$-amyloid precursor protein is inhibited by zinc in Alzheimer's disease. Cell 2010;142:857-67.

25. Priller Ch, Bauer T, Mitteregger G, Krebs B, Kretzschmar HA, Herms J. Synapse formation and function is modulated by the amyloid precursor protein. J Neurosci 2006;26:7212-21.

26. Turner PR, O'Connor K, Tate WP, Abraham WC. Roles of amyloid precursor protein and its fragments in regulating neural activity, plasticity and memory. Prog Neurobiol 2003;70:1-32.

27. Beadling C, Smith KA. DNA array analysis of interleukin-2-regulated immediate/early genes. Med Immunol 2002;1:2.

28. Behrendt N. The urokinase receptor (UPAR) and the UPAR-associated protein (UPARAP/Endo180): membrane proteins engaged in matrix turnover during tissue remodelling. Biol Chem 2004;385:103-36.

29. Van der Rest M, Garrone R. Collagen family of proteins. Fed Am Soc Exp Biol J 1991;5:2814-23.

30. Nishi Y, Uchiyama S, Doi M, Nishiuchi Y, Nakazawa T, Ohkubo T, Kobayashi Y. Different effects of 4-hydroxyproline and 4-fluoroproline on the stability of collagen triple helix. Biochemistry 2005;44:6034-42.

31. Riikonen T, Westermarck J, Koivisto L, Broberg A, Kähäri VM, Heino J. Integrin alpha 2 beta 1 is a positive regulator of collagenase (MMP-1) and collagen alpha 1(I) gene expression. J Bio Chem 1995;270:13548-52.

32. Waddington RJ, Roberts HC, Sugars RV, Schönherr E. Differential roles for small leucine-rich proteoglycans in bone formation. Eur Cell and Mater 2003;6:12-21.

33. Dafforn TR, Della M, Miller AD. The molecular interactions of heat shock protein 47 (Hsp47) and their implications for collagen biosynthesis. J Biol Chem 2001;276:49310-9.

34. Roach $\mathrm{H}$. Why does bone matrix contain non-collagenous proteins? The possible roles of osteocalcin, osteonectin, osteopontin and bone sialoprotein in bone mineralization and resorption. Cell Biol Int 1994;18:617-28.

35. Applied Biosystems: A guide to high resolution melting (HRM) analysis (2010). http://tools.thermofisher.com/content/sfs/manuals/cms_070283.pdf

36. Hrušková L, Mařík I, Mazurová S, Martásek P, Mazura I. COL1A2 gene analysis in a Czech osteogenesis imperfecta patient: a candidate novel mutation in a patient affected by osteogenesis imperfecta type 3. Advances in Genomics and Genetics 2015;5:275-81. 